\title{
COMMON CYCLIC VECTORS FOR NORMAL OPERATORS
}

\author{
WILLIAM T. ROSS AND WARREN R. WOGEN
}

\begin{abstract}
If $\mu$ is a finite compactly supported measure on $\mathbb{C}$, then the set $S_{\mu}$ of multiplication operators $M_{\phi}: L^{2}(\mu) \rightarrow L^{2}(\mu), M_{\phi} f=\phi f$, where $\phi \in L^{\infty}(\mu)$ is injective on a set of full $\mu$ measure, is the complete set of cyclic multiplication operators on $L^{2}(\mu)$. In this paper, we explore the question as to whether or not $S_{\mu}$ has a common cyclic vector.
\end{abstract}

\section{INTRODUCTION}

A bounded linear operator $S$ on a separable Hilbert space $\mathcal{H}$ is cyclic with cyclic vector $f$ if the closed linear span of $\left\{S^{n} f: n=0,1,2, \cdots\right\}$ is equal to $\mathcal{H}$. For many cyclic operators $S$, especially when $S$ acts on a Hilbert space of functions, the description of the cyclic vectors is well known. Some examples: When $S$ is the multiplication operator $f \rightarrow x f$ on $L^{2}[0,1]$, the cyclic vectors $f$ are the $L^{2}$ functions which are non-zero almost everywhere. When $S$ is the forward shift $f \rightarrow z f$ on the classical Hardy space $H^{2}$, the cyclic vectors are the outer functions [10, p. 114] (Beurling's theorem). When $S$ is the backward shift $f \rightarrow(f-f(0)) / z$ on $H^{2}$, the cyclic vectors are the non-pseudocontinuable functions $[9,16]$.

For a collection $\mathcal{S}$ of operators on $\mathcal{H}$, we say that $\mathcal{S}$ has a common cyclic vector $f$, if $f$ is a cyclic vector for every $S \in \mathcal{S}$. Earlier work of the second author [19] showed that the set of co-analytic Toeplitz operators $T_{\bar{\phi}}$, with non-constant symbol $\phi$, on $H^{2}$ has a common cyclic vector. This line of research was pursued by several others culminating in a result of Bourdon and Shapiro [4] which says that for any reproducing kernel Hilbert space of analytic functions on a planar domain $\Omega$ (Bergman space, Dirichlet space, Hardy space, etc.), the set of adjoints $M_{\phi}^{*}$ of multiplication operators, where $\phi$ is a non-constant multiplier, has a common cyclic vector.

In this paper, we will consider common cyclic vectors for normal multiplication operators. To set this up, we first review some classical results about normal operators. The interested reader can consult $[5,8]$ for further information. For our separable Hilbert space $\mathcal{H}$, let $\mathcal{B}(\mathcal{H})$ denote the bounded linear operators on $\mathcal{H}$. The weak-operator topology on $\mathcal{B}(\mathcal{H})$ (denoted by WOT) is the topology on $\mathcal{B}(\mathcal{H})$ given by the family of semi-norms

$$
\rho_{f, g}(S):=|\langle S f, g\rangle|, \quad f, g \in \mathcal{H} .
$$

We say an algebra $\mathcal{A} \subset \mathcal{B}(\mathcal{H})$ is cyclic if there is a vector $f \in \mathcal{H}$ such that $\{A f$ : $A \in \mathcal{A}\}$ is dense in $\mathcal{H}$. If $N \in \mathcal{B}(\mathcal{H})$ is normal $\left(N^{*} N=N N^{*}\right)$, let $\mathcal{W}(N)$ denote the WOT closed linear span of $\left\{N^{k}: k=0,1, \cdots\right\}$. Note that $N$ is cyclic if and only if $\mathcal{W}(N)$ is cyclic. Let $\mathcal{W}^{*}(N)$ be the (abelian) von Neumann algebra generated by $N$, i.e., $\mathcal{W}^{*}(N)$ is the WOT closed linear span of $\left\{N^{k} N^{* l}: l, k=\right.$ $0,1,2, \cdots\}$. A normal operator $N \in \mathcal{B}(\mathcal{H})$ is $*$-cyclic if the algebra $\mathcal{W}^{*}(N)$ is cyclic. 
Finally, recall that $\{N\}^{\prime}=\{A \in \mathcal{B}(\mathcal{H}): A N=N A\}$, the 'commutant' of $N$, coincides with $\mathcal{W}^{*}(N)^{\prime}$ (the operators that commute with everything in $\mathcal{W}^{*}(N)$ ) and always contains $\mathcal{W}^{*}(N)$. The following theorem is standard in the theory of normal operators.

Theorem 1.1. For a normal operator $N \in \mathcal{B}(\mathcal{H})$, the following are equivalent.

(1) $N$ is cyclic.

(2) $N$ is $*$-cyclic.

(3) $\mathcal{W}^{*}(N)$ is maximal abelian, that is to say, $\mathcal{W}^{*}(N)^{\prime}=\mathcal{W}^{*}(N)$.

(4) There is a finite, positive, Borel measure $\mu$ on the spectrum $\sigma(N)$ so that $N$ is unitarily equivalent to $M_{z}: L^{2}(\mu) \rightarrow L^{2}(\mu), M_{z} f=z f$.

Throughout this paper, all measures will be positive, finite, compactly supported, Borel measures on $\mathbb{C}$. With this assumption, $L^{2}(\mu)$ is a separable Hilbert space. The equivalence of (2), (3), and (4) is part of the spectral theory for normal operators [8, Ch. 2]. The equivalence of (1) and (2) is an elegant theorem of Bram [6, p. 232]. In general, the cyclic vectors for a normal operator will be a proper subset of the *-cyclic vectors. However, if $N$ is 'reductive' $\left(W(N)=W^{*}(N)\right)$, then each $*$-cyclic vector will be cyclic.

Since (1) and (4) are equivalent in Theorem 1.1, we consider the algebra

$$
\mathcal{A}_{\mu}:=\left\{M_{\phi}: L^{2}(\mu) \rightarrow L^{2}(\mu), M_{\phi} f=\phi f: \phi \in L^{\infty}(\mu)\right\},
$$

where $\mu$ is a measure on $\mathbb{C}$. Some basic facts about $M_{\phi}$ and $\mathcal{A}_{\mu}$ are as follows.

Proposition 1.2. For $M_{\phi} \in \mathcal{A}_{\mu}$,

(1) $M_{\phi}$ is normal with $M_{\phi}^{*}=M_{\bar{\phi}}$.

(2) $\left\|M_{\phi}\right\|=\|\phi\|_{\infty}$.

(3) $\sigma\left(M_{\phi}\right)$ is equal to $\bigcap\left\{\phi(A)^{-}\right.$: A measurable, $\left.\mu\left(A^{c}\right)=0\right\}$, often called the 'essential range' of $\phi$.

(4) $\left\{M_{z}\right\}^{\prime}=\mathcal{A}_{\mu}=W^{*}\left(M_{z}\right)$. Moreover, the map $\phi \rightarrow M_{\phi}$ is an isomorphism and a homeomorphism between $\left(L^{\infty}(\mu)\right.$, weak $\left.-*\right)$ and $\left(\mathcal{A}_{\mu}, W O T\right)$.

Concerning $*$-cyclic vectors, we can use the above discussion to say the following.

Proposition 1.3. For $\phi \in L^{\infty}(\mu)$, the following are equivalent:

(1) $M_{\phi}$ is $*$-cyclic.

(2) $\phi$ is injective on a set of full measure.

(3) $\phi$ is a weak-* generator of $L^{\infty}(\mu)$ in the sense that the weak-* closed linear span of $\left\{\phi^{n} \bar{\phi}^{k}: k, n=0,1, \cdots\right\}$ is $L^{\infty}(\mu)$.

(4) $\mathcal{W}^{*}\left(M_{\phi}\right)=\mathcal{A}_{\mu}$.

Furthermore, $f \in L^{2}(\mu)$ is $*$-cyclic for $M_{\phi}$ if and only if $f \neq 0 \mu$-almost everywhere.

Let $L_{i}^{\infty}(\mu)$ be the set of $\phi \in L^{\infty}(\mu)$ that are injective on a set of full measure. Also define $S_{\mu}:=\left\{M_{\phi}: \phi \in L_{i}^{\infty}(\mu)\right\}$ and note that $S_{\mu}$ has a common $*$-cyclic vector (any $f \in L^{2}(\mu)$ that is non-zero $\mu$-almost everywhere will do) and in fact, the common $*$-cyclic vectors are dense. Also observe that the set $S_{\mu}^{r}:=\left\{M_{\phi}\right.$ : $\left.\phi \in L_{i}^{\infty}(\mu), W\left(M_{\phi}\right)=W^{*}\left(M_{\phi}\right)\right\}$ has a common cyclic vector. Our common cyclic vector problem, and the focus of this paper, is the following.

Question 1.4. Does $S_{\mu}$ have a common cyclic vector? 
S. Seubert kindly informed us that the answer yes when the measure $\mu$ is atomic. In fact, results in a paper of Sibilev [17] ([14] has a related result) essentially characterize the common cyclic vectors for $S_{\mu}$ when $\mu$ is atomic. We outline a short proof of this result (Theorem 2.3) for completeness and to suggest some further directions.

We will show, for a general measure $\mu$, decomposed as $\mu=\mu_{d}+\mu_{c}$, with $\mu_{d}$ discrete and $\mu_{c}$ continuous [12, p. 334], that $S_{\mu}$ has a common cyclic vector only when $\mu_{c}=0$ (Theorem 3.4). Using the theory of Lebesgue spaces, it is enough to consider the measure $\mu=m$, normalized Lebesgue measure on the unit circle $\mathbb{T}$. We will show that $S_{m}$ has no common cyclic vectors (Proposition 3.2) and thus one is led to consider whether or not natural subsets of $S_{m}$ have common cyclic vectors. Theorem 4.1 is such a result.

\section{Atomic measures}

Suppose $\mu$ is purely atomic, that is,

$$
d \mu=\sum_{n=1}^{\infty} a_{n} \delta_{z_{n}}
$$

where $\left(a_{n}\right)_{n \geqslant 1}$ is a summable sequence of positive numbers and $\delta_{z_{n}}$ is the unit point mass at $z_{n}$. For $\phi \in L_{i}^{\infty}(\mu)$, observe that $M_{\phi} \chi_{z_{n}}=\phi\left(z_{n}\right) \chi_{z_{n}}$, where $\chi_{z_{n}}$ is the characteristic function for the singleton $\left\{z_{n}\right\}$. Thus the operator $M_{\phi}$ has a spanning set of eigenvectors $\left\{\chi_{z_{n}}: n=1,2, \cdots\right\}$ corresponding to distinct eigenvalues (since $\phi$ is injective). We claim that

$$
h:=\sum_{n=1}^{\infty} 2^{-n} \chi_{z_{n}}
$$

is a common cyclic vector for $S_{\mu}$

Theorem 2.3. For a purely atomic measure $\mu, S_{\mu}$ has a common cyclic vector.

The key to proving Theorem 2.3 is a function theory result of Beurling. The set up is as follows: Suppose that $\left(c_{n}\right)_{n \geqslant 1}$ is a sequence of complex numbers satisfying

$$
\varlimsup_{n \rightarrow \infty} \sqrt[n]{\left|c_{n}\right|}<1
$$

and $\left(a_{n}\right)_{n \geqslant 1}$ is a bounded sequence of distinct complex numbers. Form the function

$$
f(z):=\sum_{n=1}^{\infty} \frac{c_{n}}{z-a_{n}}
$$

(functions of this type are often called 'Borel series' or sometimes 'Denjoy-Wolff' series) and note that $f$ is analytic off $\left(a_{n}\right)_{n \geqslant 1}^{-}$. Note also that $\mathbb{C} \backslash\left(a_{n}\right)_{n \geqslant 1}^{-}$might be a disconnected set. The following is a consequence of a more general result of Beurling [3, Théorème II] (see also [16]).

Lemma 2.5 (Beurling). If the function in eq.(2.4) vanishes on an open subset of $\mathbb{C} \backslash\left(a_{n}\right)_{n \geqslant 1}^{-}$, then $c_{n}=0$ for all $n$.

We are now ready for the proof of Theorem 2.3. With $d \mu$ and $h$ defined in eq.(2.1) and eq.(2.2), let $\phi \in L_{i}^{\infty}(\mu)$. If $g \in L^{2}(\mu)$ satisfies $\left\langle M_{\phi}^{N} h, g\right\rangle=0$ for every 
$N=0,1,2, \cdots$, then

$$
0=\sum_{n=1}^{\infty} \phi\left(z_{n}\right)^{N} h\left(z_{n}\right) \overline{g\left(z_{n}\right)} a_{n}=\sum_{n=1}^{\infty} \phi\left(z_{n}\right)^{N} 2^{-n} \overline{g\left(z_{n}\right)} a_{n}, \quad N=0,1,2, \cdots .
$$

Notice that

$$
\sum_{n=1}^{\infty}\left|g\left(z_{n}\right)\right| a_{n}=\int|g| d \mu \leqslant C\left[\int|g|^{2} d \mu\right]^{1 / 2}<\infty
$$

and so, in particular, $\left(\overline{g\left(z_{n}\right)} a_{n}\right)_{n \geqslant 1}$ is a bounded sequence.

For $|z|>A:=\sup \left\{\left|\phi\left(z_{n}\right)\right|: n=1,2, \cdots\right\}$,

$$
0=\sum_{N=0}^{\infty} \frac{1}{z^{N}} \sum_{n=1}^{\infty} \phi\left(z_{n}\right)^{N} 2^{-n} \overline{g\left(z_{n}\right)} a_{n}
$$

which, after reversing the order to summation, yields

$$
0=\sum_{n=1}^{\infty} 2^{-n} \overline{g\left(z_{n}\right)} a_{n} \sum_{N=0}^{\infty} \frac{\phi\left(z_{n}\right)^{N}}{z^{N}}=\sum_{n=1}^{\infty} \frac{2^{-n} \overline{g\left(z_{n}\right)} a_{n}}{1-\phi\left(z_{n}\right) / z}=0, \quad|z|>A .
$$

Now appeal to Beurling's result, Lemma 2.5 (note that

$$
\varlimsup_{n \rightarrow \infty} \sqrt[n]{\left|2^{-n} \overline{g\left(z_{n}\right)} a_{n}\right|} \leqslant 1 / 2
$$

and the $\phi\left(z_{n}\right)$ 's are distinct, since $\phi$ is injective) to conclude that $g\left(z_{n}\right)=0$ for all $n$ and so, since $g$ vanishes on a carrier of $\mu, g$ is the zero function. Since $h$ does not depend on $\phi \in L_{i}^{\infty}(\mu), h$ is a common cyclic vector for $S_{\mu}$.

\section{Measures With a CONTINuOUs part}

We will now show that $S_{\mu}$ does not have a common cyclic vector whenever $\mu$ has a non-trivial continuous part. Before we do though, we recall Szegö's theorem [13, p. 49].

Theorem 3.1 (Szegö). For a positive measure $\mu$ on $\mathbb{T}$ with Lebesgue decomposition, with respect to normalized Lebesgue measure $m$ on the circle, $d \mu=h d m+d \sigma$, where $h \in L^{1}(m)$ and $\sigma \perp m$,

$$
\inf _{p \in \mathcal{P}, p(0)=0} \int|1-p|^{2} d \mu=\exp \left[\int_{\mathbb{T}} \log h d m\right],
$$

where $\mathcal{P}$ is the set of analytic polynomials.

We first consider the case when $\mu=m$.

Proposition 3.2. $S_{m}$ does not have a common cyclic vector.

Proof. Suppose that $f \in L^{2}(m)$ is cyclic for $M_{z}$. As a consequence of Szegö's theorem (Theorem 3.1),

$$
\int_{\mathbb{T}} \log |f| d m=-\infty .
$$

For $n \in \mathbb{N}$, let

and

$$
E_{n}:=\left\{e^{i \theta}: \frac{1}{2^{n}}<\left|f\left(e^{i \theta}\right)\right| \leqslant \frac{1}{2^{n-1}}\right\}
$$

$$
E_{0}:=\left\{e^{i \theta}:\left|f\left(e^{i \theta}\right)\right|>1\right\} .
$$


We can assume, without loss of generality (perhaps multiplying by a constant, which does not change cyclicity), that $m\left(E_{0}\right)>0$. On the set $E_{n}$ note that $\log \left|f\left(e^{i \theta}\right)\right| \approx$ $-n$.

We will now construct our $\phi \in L_{i}^{\infty}(m)$ for which $f$ is not cyclic for $M_{\phi}$. To make the construction conceptually easier, we will equate $\mathbb{T}$ with $[0,2 \pi]$ and $m$ with Lebesgue measure on $[0,2 \pi]$. For $x \in E_{1}$, let

and note that

$$
\phi(x)=\int_{0}^{x} \chi_{E_{1}}(s) d s
$$

and

$$
\frac{d(m \circ \phi)}{d m} \mid E_{1}=1
$$

For $x \in E_{2}$, let

$$
F_{1}:=\phi\left(E_{1}\right)=\left[0, m\left(E_{1}\right)\right] .
$$

and note that

$$
\phi(x)=m\left(E_{1}\right)+\frac{1}{2} \int_{0}^{x} \chi_{E_{2}}(s) d s
$$

and

$$
\frac{d(m \circ \phi)}{d m} \mid E_{2}=\frac{1}{2}
$$

In general, for $x \in E_{n}$, let

$$
F_{2}:=\phi\left(E_{2}\right)=\left[m\left(E_{1}\right), m\left(E_{1}\right)+\frac{1}{2} m\left(E_{2}\right)\right] .
$$

$$
\phi(x)=\sum_{j=1}^{n-1} \frac{1}{j} m\left(E_{j}\right)+\frac{1}{n} \int_{0}^{x} \chi_{E_{n}}(s) d s
$$

and note that

$$
\frac{d(m \circ \phi)}{d m} \mid E_{n}=\frac{1}{n}
$$

and

$$
F_{n}:=\phi\left(E_{n}\right)=\left[\sum_{j=1}^{n-1} \frac{1}{j} m\left(E_{j}\right), \sum_{j=1}^{n} \frac{1}{j} m\left(E_{j}\right)\right] .
$$

Finally, for $x \in E_{0}$, let

$$
\phi(x)=\sum_{n=1}^{\infty} \frac{1}{n} m\left(E_{n}\right)+\frac{1}{m\left(E_{0}\right)}\left[2 \pi-\sum_{n=1}^{\infty} \frac{1}{n} m\left(E_{n}\right)\right] \int_{0}^{x} \chi_{E_{0}}(s) d s
$$

and so

$$
F_{0}:=\phi\left(E_{0}\right)=\left[\sum_{n=1}^{\infty} \frac{1}{n} m\left(E_{n}\right), 2 \pi\right] .
$$

By construction, $\phi \in L_{i}^{\infty}(m)$ and for $n=1,2, \cdots$,

$$
\begin{gathered}
\frac{d(m \circ \phi)}{d m} \mid E_{n}=\frac{1}{n}, \\
\frac{d\left(m \circ \phi^{-1}\right)}{d m} \mid F_{n}=n .
\end{gathered}
$$

Also note that $m$ and $m \circ \phi^{-1}$ are mutually absolutely continuous and so

$$
U g:=\sqrt{\frac{d\left(m \circ \phi^{-1}\right)}{d m}} \cdot\left(g \circ \phi^{-1}\right)
$$


is a unitary map on $L^{2}(m)$ with $U M_{\phi} U^{-1}=M_{z}$.

Let us now argue that $U f$ is not cyclic for $M_{z}$. To do this, apply Szegö's theorem (Theorem 3.1) to the function

to get

$$
U f=\sqrt{\frac{d\left(m \circ \phi^{-1}\right)}{d m}} \cdot\left(f \circ \phi^{-1}\right)
$$

$\int_{0}^{2 \pi} \log \left[\sqrt{\frac{d\left(m \circ \phi^{-1}\right)}{d m}}\left|f \circ \phi^{-1}\right|\right] d \theta=\sum_{n=0}^{\infty} \int_{F_{n}}\left[\log \sqrt{\frac{d\left(m \circ \phi^{-1}\right)}{d m}}+\log \left|f \circ \phi^{-1}\right|\right] d \theta$.

On the set $F_{n}, \log \left|f \circ \phi^{-1}\right| \approx-n$ and so

$$
\sum_{n=1}^{\infty} \int_{F_{n}} \log \left|f \circ \phi^{-1}\right| d \theta \approx \sum_{n=1}^{\infty}-n\left(\frac{1}{n} m\left(E_{n}\right)\right)
$$

which converges. Recall that,

$$
\frac{d\left(m \circ \phi^{-1}\right)}{d m} \mid F_{n}=n
$$

and so

$$
\sum_{n=1}^{\infty} \int_{F_{n}} \log \sqrt{\frac{d\left(m \circ \phi^{-1}\right)}{d m}} d \theta=\sum_{n=1}^{\infty}\left(\frac{1}{2} \log n\right) \cdot\left(\frac{1}{n} m\left(E_{n}\right)\right)
$$

which also converges. Thus $U f$ is log-integrable and so by Szegö's theorem, $U f$ is not a cyclic vector for $M_{z}$.

Finally notice that from the above argument and the identity $U M_{\phi} U^{-1}=M_{z}$ that $U^{-1} U f=f$ is not cyclic for $M_{\phi}$.

Remark 3.3. The above proof in fact shows that $M_{\phi}$ is unitarily equivalent to $M_{z}$.

Theorem 3.4. If $\mu_{c} \neq 0$, then $S_{\mu}$ does not have a common cyclic vector.

Proof. Decompose the measure $\mu$ as $\mu=\mu_{d}+\mu_{c}$, where $\mu_{d}$ is discrete and $\mu_{c}$ is continuous in that $\mu_{c}(\{z\})=0$ for all $z \in \mathbb{C}\left[12, \mathrm{p}\right.$. 334]. The measures $\mu_{d}$ and $\mu_{c}$ are carried by the sets $E_{d}$ and $E_{c}$ respectively, which we can assume to be disjoint. The mapping $f \rightarrow f\left|E_{d}+f\right| E_{c}$ defines a unitary map between $L^{2}(\mu)$ and $L^{2}\left(\mu_{d}\right) \oplus L^{2}\left(\mu_{c}\right)$. If, for $\phi \in L^{\infty}(\mu),\left(M_{\phi}, L^{2}(\mu)\right)$ is cyclic with cyclic vector $f$, then a routine observation yields that both $\left(M_{\phi}, L^{2}\left(\mu_{d}\right)\right)$ and $\left(M_{\phi}, L^{2}\left(\mu_{c}\right)\right)$ must also be cyclic with cyclic vectors $f \mid E_{d}$ and $f \mid E_{c}$ respectively. We will complete the proof by showing that $S_{\mu_{c}}$ does not have a common cyclic vector.

To this end, assume that $\mu=\mu_{c}$ and let $X$ be the support of $\mu$. Note that we can also assume that $\|\mu\|=1$. From a classical theorem on Lebesgue spaces appearing in [11] (see also [8, p. 292]), there are Borel sets $\Delta_{1} \subset X, \Delta_{2} \subset \mathbb{T}$ and a bijection $\tau: \Delta_{1} \rightarrow \Delta_{2}$ such that (i) $\mu\left(X \backslash \Delta_{1}\right)=0=m\left(\mathbb{T} \backslash \Delta_{2}\right)$; (ii) A subset $\Delta \subset \Delta_{1}$, is $\mu$-measurable if and only if $\tau(\Delta)$ is Lebesgue measurable; (iii) $\mu(\Delta)=m(\tau(\Delta))$, i.e., $\tau$ is measure preserving.

One can check that $U f:=f \circ \tau$ defines a unitary operator from $L^{2}(m)$ to $L^{2}(\mu)$ and that for each $\phi \in L^{\infty}(m)$,

$$
U\left(M_{\phi}, L^{2}(m)\right) U^{-1}=\left(M_{\phi \circ \tau}, L^{2}(\mu)\right)
$$

This makes the map $M_{\phi} \rightarrow U M_{\phi} U^{-1}$ a spatial isomorphism between $\mathcal{A}_{m}$ and $\mathcal{A}_{\mu}$. Since $S_{m}$ does not have a common cyclic vector (Proposition 3.2), $S_{\mu}$ does not have a common cyclic vector. 
Remark 3.5. (1) We mention that using Lebesgue spaces, one can give an alternate construction of the map $\phi$ in the proof of Proposition 3.2. The details are left to the interested reader.

(2) In the proof of Theorem 3.4, we used the fact that if $f$ is cyclic for $\left(M_{\phi}, L^{2}(\mu)\right)$, then $f \mid E_{d}$ is cyclic for $\left(M_{\phi}, L^{2}\left(\mu_{d}\right)\right)$ and $f \mid E_{c}$ is cyclic for $\left(M_{\phi}, L^{2}\left(\mu_{c}\right)\right)$. The converse of this is not true in that the sum of a cyclic vector for $M_{\phi}$ on $L^{2}\left(\mu_{d}\right)$ and a cyclic vector for $M_{\phi}$ on $L^{2}\left(\mu_{c}\right)$ may not be cyclic for $M_{\phi}$ on $L^{2}(\mu)($ see $[2$, Ex. 9])

\section{A positive COMmon CYCLIC VECTOR RESUlt}

Let $\mathcal{C}$ be the set of $\phi \in C^{1+\varepsilon}(\mathbb{T})$, for some $\varepsilon>0$, such that $\phi$ is injective, except possibly for a finite number of points, and such that

$$
\frac{d}{d \theta} \phi\left(e^{i \theta}\right) \neq 0
$$

for all $e^{i \theta}$. Let us abuse notation slightly and let

$$
\phi^{\prime}\left(e^{i \theta}\right):=\frac{d}{d \theta} \phi\left(e^{i \theta}\right) .
$$

Our common cyclic vector for $\left\{\left(M_{\phi}, L^{2}(m)\right): \phi \in \mathcal{C}\right\}$ will be any vector $f \in L^{2}(m)$ that is non-zero $m$-a.e. and which also satisfies

$$
\int_{J} \log |f| d m=-\infty
$$

for every arc $J \subset \mathbb{T}$. An example of such a function is the following: For a sequence $\left(a_{n}\right)_{n \geqslant 1}$ of points that are dense in $\mathbb{T}$, define

$$
f\left(e^{i \theta}\right):=\exp \left(-\sum_{n=1}^{\infty} \frac{2^{-n}}{\left|e^{i \theta}-a_{n}\right|}\right)
$$

and note that $f$ is bounded, non-zero almost everywhere, and not log-integrable on any arc of the circle.

Theorem 4.1. A function $f \in L^{2}(m)$ is cyclic for every $M_{\phi}, \phi \in \mathcal{C}$, if and only if $f$ is non-zero $m$-a.e. and $f$ is not log-integrable on any arc of the circle.

For a measure $\mu$, let $P^{2}(\mu)$ be the closure of the analytic polynomials in $L^{2}(\mu)$. The proof of our positive cyclic vector result (Theorem 4.1) needs a few preliminaries.

Proposition 4.2. For a measure $\mu$, let $g \in L^{2}(\mu)$ be non-zero $\mu$-a.e. If $P^{2}(\nu)=$ $L^{2}(\nu)$, where $d \nu=|g|^{2} d \mu$, then $g$ is a cyclic vector for $\left(M_{z}, L^{2}(\mu)\right)$.

Proof. Let $V$ denote the closed linear span of $\left\{M_{z}^{n} g: n=0,1,2, \cdots\right\}$. The equality $P^{2}(\nu)=L^{2}(\nu)$ implies that $f g \in V$ for all functions $f$ that are continuous on the support of $\mu$. If $h \in L^{2}(\mu)$ such that

$$
\int f g \bar{h} d \mu=0
$$

for all such continuous $f$, then $g \bar{h} d \mu$ is the zero measure. But since $g$ is non-zero $\mu$-a.e., this means that $h$ is zero $\mu$-a.e. and so $V=L^{2}(\mu)$. 
For fixed $\phi \in \mathcal{C}$, the set $\phi(\mathbb{T}):=\Gamma$ is a $C^{1+\varepsilon}$ planar curve which intersects itself a finite number of times. The operator

$$
U h=\left|\phi^{\prime} \circ \phi^{-1}\right|^{-1 / 2} \cdot\left(h \circ \phi^{-1}\right)
$$

is a unitary operator from $L^{2}(m)$ to $L^{2}(\Gamma, d s)$, where $d s$ denotes arc length measure on $\Gamma$, with

$$
U^{*}\left(M_{z}, L^{2}(\Gamma, d s)\right) U=\left(M_{\phi}, L^{2}(m)\right)
$$

Also needed here are the following two generalizations of Szegö's theorem. The first is obtained from the classical version of Szegö's theorem (Theorem 3.1) by a conformal mapping.

Theorem 4.4. Let $\Omega$ be a Jordan domain and $\mu$ a measure supported in $\partial \Omega$. If $\mu=\mu_{a}+\mu_{s}$ is the Lebesgue decomposition of $\mu$ with respect to $\omega_{\Omega}$, i.e., $\mu_{a} \ll \omega_{\Omega}$, $\mu_{s} \perp \omega_{\Omega}$, then $P^{2}(\mu)=L^{2}(\mu)$ if and only if

$$
\int_{\partial \Omega} \log \left(\frac{d \mu_{a}}{d \omega_{\Omega}}\right) d \omega_{\Omega}=-\infty
$$

The second version is due to Akeroyd [1, 2] (see [18] for a related result) which we state verbatim.

Proposition 4.5. Let $K$ be a compact, connected, subset of $\mathbb{C}$, and let $\gamma$ be a Jordan arc that (except for its end points which are in $K$ ) lies in the unbounded component of $K^{c}$ and let $\mu$ be a measure supported in $\gamma \cup K$. Let $\Omega$ be the bounded component of $(\gamma \cup K)^{c}$ whose boundary contains $\gamma$, and let

$$
d \mu \mid \gamma=h d \omega_{\Omega}+d \mu_{s}
$$

be the Lebesgue decomposition of $\mu \mid \gamma$, with respect to $\omega_{\Omega}$ on $\gamma$. If

$$
\int_{\gamma} \log h d \omega_{\Omega}=-\infty
$$

then $\chi_{\gamma} \in P^{2}(\mu)$.

Let us say a few words about these two results. First, $\omega_{\Omega}$ is harmonic measure on $\partial \Omega$ evaluated at some point in $\Omega$. Secondly, in our application of this theorem (see below), we will be taking $\Omega$ to be one of the bounded components of $\Gamma^{c}$ (where $\Gamma=\phi(\mathbb{T}), \phi \in \mathcal{C})$ in which case $\partial \Omega$ is a piecewise smooth curve.

Lemma 4.6. If $J$ is a closed $C^{1+\varepsilon}$ sub-arc of $\partial \Omega$ which does not contain any of the 'crossing points' of $\Omega=\phi(\mathbb{T})$, and $\mu$ is a measure on $J$, then

(1) the function $\frac{d s}{d \omega_{\Omega}}$ is bounded above and below on $J$,

(2) there are $c_{1}, c_{2}>0$ so that

$$
c_{1} \omega_{\Omega}(A) \leqslant s(A) \leqslant c_{2} \omega_{\Omega}(A)
$$

for all Borel subsets $A$ of $J$,

(3)

if and only if

$$
\int_{J} \log \left(\frac{d \mu}{d \omega_{\Omega}}\right) d \omega_{\Omega}=-\infty
$$

$$
\int_{J} \log \left(\frac{d \mu}{d s}\right) d s=-\infty .
$$


Proof. If $\psi: \mathbb{D} \rightarrow \Omega$ is the Riemann map with $\psi(0)=a$ (where $a \in \Omega$ is the evaluation point of $\left.\omega_{\Omega}\right)$ and $\psi^{\prime}(0)>0$, then $\theta \rightarrow \psi\left(e^{i \theta}\right)$ is a parameterization of $\partial \Omega$. Moreover, if $J$ is any smooth $\operatorname{arc}$ of $\partial \Omega$, then, by using the hypothesis that $J$ is in fact $C^{1+\varepsilon}$, we obtain that $\psi^{\prime}$ extends to be a continuous function on $\psi^{-1}(J)[15$, p. 48] (actually, one proves a local version of the theorem stated in the reference by means of a conformal mapping). From this, we conclude that $\left|\psi^{\prime}\right|$ is bounded above and below on $\psi^{-1}(J)$. It is a standard fact (see for example [7, p. 302]) that the arc-length measure of a set $A \subset \partial \Omega$ is

$$
\int_{\psi^{-1}(A)}\left|\psi^{\prime}\left(e^{i \theta}\right)\right| d \theta
$$

and the harmonic measure of $A$ is

$$
\omega_{\Omega}(A)=\int_{\psi^{-1}(A)} \frac{d \theta}{2 \pi} .
$$

Thus, for subsets $A$ of $J, \omega_{\Omega}(A)$ is comparable to the arc length measure on $J$ and moreover on $J$,

$$
\frac{d s}{d \omega_{\Omega}}=\frac{1}{2 \pi}\left|\psi^{\prime}\left(\psi^{-1}\right)\right|
$$

This proves statements (1) and (2) of the lemma.

Finally, notice that

$$
\int_{J} \log \left(\frac{d \mu}{d \omega_{\Omega}}\right) d \omega_{\Omega}=\int_{J} \log \left(\frac{d \mu}{d s} \frac{d s}{d \omega_{\Omega}}\right) d \omega_{\Omega} .
$$

Combine this with the first two statements of the lemma, to prove statement (3).

Proof of Theorem 4.1: One direction of Theorem 4.1 is relatively easy to prove. Indeed, if $f$ is cyclic for every operator in $\left\{M_{\phi}: \phi \in \mathcal{C}\right\}$, then certainly $f$ is cyclic for $M_{z}$ on $L^{2}(m)$ and so $f$ is non-zero $m$-a.e. To show that $f$ is not log-integrable on every arc $J$ of the circle, we let $\phi \in \mathcal{C}$ map the arc $J$ onto a smooth closed curve $\Gamma_{1}$ and such that $\phi(\mathbb{T})$ intersects itself only once (at the endpoints of $\Gamma_{1}$ ). We let $\Gamma_{2}=\phi(\mathbb{T} \backslash J)$ and notice that the map $F \rightarrow F\left|\Gamma_{1}+F\right| \Gamma_{2}$ is unitary from $L^{2}\left(\Gamma_{1} \cup \Gamma_{2}, d s\right)$ to $L^{2}\left(\Gamma_{1}, d s\right) \oplus L^{2}\left(\Gamma_{2}, d s\right)$ that makes

$$
\left(M_{z}, L^{2}\left(\Gamma_{1} \cup \Gamma_{2}, d s\right)\right) \cong\left(M_{z}, L^{2}\left(\Gamma_{1}, d s\right)\right) \oplus\left(M_{z}, L^{2}\left(\Gamma_{2}, d s\right)\right) .
$$

Since $f$ must be cyclic for this particular $M_{\phi}$, then $g=U f$ (recall the definition of $U$ from eq.(4.3)) must be cyclic for $\left(M_{z}, L^{2}\left(\Gamma_{1} \cup \Gamma_{2}, d s\right)\right)$ and hence $g \mid \Gamma_{1}$ must be cyclic for $\left(M_{z}, L^{2}\left(\Gamma_{1}, d s\right)\right)$. But then $P^{2}\left(|g|^{2} d s\right)=L^{2}\left(|g|^{2} d s\right)$ and so, by Theorem 4.4 and Lemma 4.6,

$$
\int_{\Gamma_{1}} \log |g| d s=-\infty .
$$

However, after a change of variables and Lemma 4.6, this integral is comparable to

$$
\int_{J} \log |f| d m \text {. }
$$

To prove the converse, we will show, for every $f \in L^{2}(m)$ which is non-zero almost everywhere and is not log-integrable on any arc of the circle, that for every $\Gamma=\phi(\mathbb{T})$, the vector $g=U f$ is cyclic for $\left(M_{z}, L^{2}(\Gamma, d s)\right)$. By Proposition 4.2, it 
suffices to show that $P^{2}(\nu)=L^{2}(\nu)$, where $d \nu=|g|^{2} d s$. Notice that $g$ is non-zero $d s$-a.e. and that for any $\operatorname{arc} \gamma \subset \Gamma$,

$$
\int_{\gamma} \log |g| d s=-\infty
$$

To see that $P^{2}(\nu)=L^{2}(\nu)$, let $\gamma$ be a closed sub-arc of $\Gamma$ that is part of the boundary of the unbounded component of $\Gamma^{c}$ and which does not contain any of the intersection points of $\Gamma$. Apply Proposition 4.5 with $K=\Gamma \backslash \gamma$ and $\mu=\nu$ (along with Lemma 4.6) to see that $\chi_{\gamma} \in P^{2}(\nu)$. But since this arc $\gamma$ was arbitrary, we obtain the decomposition

$$
P^{2}(\nu)=L^{2}\left(\nu \mid \Gamma_{0}\right) \oplus P^{2}\left(\nu \mid\left(\Gamma \backslash \Gamma_{0}\right)\right),
$$

where $\Gamma_{0}$ is the portion of $\Gamma$ which is the boundary of the unbounded component of $\Gamma^{c}$. Now apply the same argument to $P^{2}\left(\nu \mid\left(\Gamma \backslash \Gamma_{0}\right)\right)$ and so on (a finite number of times) to see that $P^{2}(\nu)=L^{2}(\nu)$.

Remark 4.7. The same proof, with the obvious changes, shows that Theorem 4.1

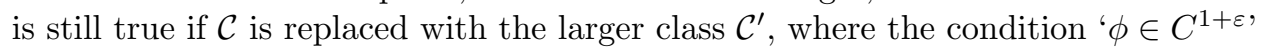
is relaxed to ' $\phi$ is piecewise $C^{1+\varepsilon}$ '. In addition, it is not hard to see that the set of common cyclic vectors for $\left\{M_{\phi}: \phi \in \mathcal{C}^{\prime}\right\}$ is norm dense in $L^{2}(m)$.

If $\mu$ is a measure for which $\mu_{c}$ is not zero, then $S_{\mu}$ does not have a common cyclic vector. What are some natural subclasses of $S_{\mu}$ with common cyclic vectors? Can one identify a maximal subclass of $S_{\mu}$ with a common cyclic vector? Note that such a maximal subclass must contain $S_{\mu}^{r}$. This maximal question is open even for $S_{m}$.

\section{REFERENCES}

1. J. Akeroyd, An extension of Szegö's theorem, Indiana Univ. Math. J. 43 (1994), no. 4, 13391347. MR 96d:30045

2. _ An extension of Szegö's theorem. II, Indiana Univ. Math. J. 45 (1996), no. 1, 241-252. MR 97h:30055

3. A. Beurling, Sur les fonctions limites quasi analytiques des fractions rationnelles, Proc. Eighth Scand. Math. Congress, Lund (1935), 199-210.

4. P. Bourdon and J. H. Shapiro, Spectral synthesis and common cyclic vectors, Michigan Math. J. 37 (1990), no. 1, 71-90. MR 91m:47039

5. J. B. Conway, A course in functional analysis, second ed., Graduate Texts in Mathematics, vol. 96, Springer-Verlag, New York, 1990. MR 91e:46001

6. _ The theory of subnormal operators, Mathematical Surveys and Monographs, vol. 36, American Mathematical Society, Providence, RI, 1991. MR 92h:47026

7. , Functions of one complex variable. II, Graduate Texts in Mathematics, vol. 159, Springer-Verlag, New York, 1995. MR 96i:30001

8. 1 , A course in operator theory, Graduate Studies in Mathematics, vol. 21, American Mathematical Society, Providence, RI, 2000. MR 2001d:47001

9. R. G. Douglas, H. S. Shapiro, and A. L. Shields, Cyclic vectors and invariant subspaces for the backward shift operator., Ann. Inst. Fourier (Grenoble) 20 (1970), no. fasc. 1, 37-76. MR $42 \# 5088$

10. P. L. Duren, Theory of $H^{p}$ spaces, Academic Press, New York, 1970. MR 42 \#3552

11. P. Halmos and J. von Neumann, Operator methods in classical mechanics. II, Ann. of Math. (2) 43 (1942), 332-350. MR 4,14e

12. E. Hewitt and K. Stromberg, Real and abstract analysis. A modern treatment of the theory of functions of a real variable, Springer-Verlag, New York, 1965. MR 32 \#5826

13. K. Hoffman, Banach spaces of analytic functions, Dover Publications Inc., New York, 1988, Reprint of the 1962 original. MR 92d:46066 
14. E. Nordgren and P. Rosenthal, A sufficient condition that an operator be cyclic, unpublished.

15. Ch. Pommerenke, Boundary behaviour of conformal maps, Grundlehren der Mathematischen Wissenschaften [Fundamental Principles of Mathematical Sciences], vol. 299, Springer-Verlag, Berlin, 1992. MR 95b:30008

16. W. T. Ross and H. S. Shapiro, Generalized analytic continuation, University Lecture Series, vol. 25, American Mathematical Society, Providence, RI, 2002. MR 2003h:30003

17. R. V. Sibilev, A uniqueness theorem for Wolff-Denjoy series, Algebra i Analiz 7 (1995), no. 1, 170-199. MR 96j:30006

18. T. Trent, Extension of a theorem of Szegö, Michigan Math. J. 26 (1979), no. 3, 373-377. MR 80j:30050

19. W. Wogen, On some operators with cyclic vectors, Indiana Univ. Math. J. 27 (1978), no. 1, 163-171. MR 57 \#7234

Department of Mathematics and Computer Science, University of Richmond, RichMOND, VIRGINIA 23173

E-mail address: wross@richmond.edu

Department of Mathematics, University of North Carolina - Chapel Hill, Chapel Hill, North Carolina, 27599

E-mail address: wrw@email.unc.edu 